\title{
Calcified Right Ventricular Fibroma in an Adult: A Case Report
}

\author{
Huanhuan $\mathrm{Gao}^{1}$, Shuai Yuan ${ }^{1}$, Zhiqiang $\mathrm{Hu}^{1}$, Zhelan Zheng ${ }^{1}$, and Shengjun $\mathrm{Wu}^{1}$ \\ ${ }^{1}$ Zhejiang University School of Medicine First Affiliated Hospital
}

June 4, 2021

\begin{abstract}
Background: Cardiac fibromas are rare benign tumors of the heart composed of fibroblasts and collagen. They are common among children and adolescents but are rarely present in adults. Case presentation: We here report the case of a fiftyseven-year-old man who complaining of a 2-year history of chest tightness at rest. Transthoracic echocardiography detected a severe calcified mass protruding outside the right ventricular anterior wall near the apex. The patient was referred for tumor resection. The calcified mass was determined to be a cardiac fibroma with postoperative histopathological examination. The patient experienced an unremarkable post-operative recovery and was discharged 8 days later. Subsequent follow-up has shown complete freedom from his troublesome symptom. Conclusions: Preoperative diagnosis with various imaging modalities and early surgery are the keys to improve prognosis of patients with cardiac fibromas.
\end{abstract}

\section{Introduction}

Cardiac fibromas are rare innocent tumors composed of fibroblasts and collagen in the myocardium, which have been regard as the second most frequent benign cardiac tumor following rhabdomyoma among children but rarely seen in adults [1,2]. Cardiac fibromas are mainly located in the ventricles or interventricular septum and present invariably as central calcification, which distinguishes them from rhabdomyomas [3]. The clinical manifestations are nonspecific, including stethalgia, arrhythmias, dyspnoeic, and even sudden death, which depend on their position and size [4]. However, it is predicted that some patients with cardiac fibroma are symptomless and found on imageological diagnosis accidentally [3]. Early surgery is the absolute key to improve outcome of symptomatic patient $[5,6]$.

\section{Case Report}

A 57-year-old man displayed chest tightness and nausea about two years, hospitalized to the First Affiliated Hospital of Zhejiang University (Hangzhou, China) in December 2020. The patient was in good condition generally, denying palpitations, syncope, chest pain and dyspnea. The blood pressure, heart rate, electrocardiogram and biochemical examinations were within the normal range. However, the right calcified cardiac border was found incidentally by chest X-ray. In addition, transthoracic echocardiography (TTE) revealed a $4.3 \mathrm{~cm} \times 0.7 \mathrm{~cm}$ area of calcification within the myocardium located at the right ventricular anterior wall near the apical but the right ventricular wall motion was normal [Figure 1a]. The diagnosis of a benign pericardial tumor was confirmed through the computed tomography (CT) and three-dimensional reconstruction imaging [Figure 1b-1c]. Intraoperative transesophageal echocardiography (TEE )identified a $3 \mathrm{cmx} 2 \mathrm{~cm}$ hyperechoic mass in the apical interventricular groove stretching to the right ventricular anterior wall [Figure 1d].

The patient proceeded the surgical excision of the tumor under extracorporeal circulation state, and median sternotomy was executed. A protruding yellowish firm mass $(3 \mathrm{~cm} \mathrm{x} 3 \mathrm{~cm} \mathrm{x2} \mathrm{cm})$ close to the left anterior descending artery branch was discovered [Figure 2a]. Finally, he elastic tumor was carefully and completely resected without damaging the left anterior descending artery[Figure $2 \mathrm{~b}]$. 
Histopathologic examination showed that the mass was a fibroma, presenting as tumorlike fibrous tissue hyperplasia with abundant collagen fibers. No evidence of mitotic figures or malignancy was observed [Figure 3]. The patient recovered well after the operation and discharged at 8 days after surgery. Follow-up echocardiography was examined every 3 months postoperative, there is no evidence of recurrence and the patient remains asymptomatic.

\section{Discussion}

Cardiac fibroma is an infrequent benign tumor in adults [2]. Sometimes, necrosis and cystic degeneration may be observed in this tumor [3,4]. Echocardiography is regarded as the preferred inspection method in general owing to the accessibility and non-invasion [2]. In addition, both CT and magnetic resonance imaging (MRI) could provide tumor localization, and identify tissue characterization and surrounding structure [2,3]. In addition, MRI can provide additional functional data. Therefore, Furthermore, cardiac MRI could further reveal the underlying pathological mechanisms and evaluate hemodynamic effects [5].

In our case, the tumor originated from the right ventricle myocardium and was nourished by the distal left anterior descending artery branch.On the whole, the calcified cardiac fibroma is firm and presented with smooth yellowish surface.

The severity of clinical manifestations depends on their position and size [4]. Cardiac fibroma hardly ever subsides naturally and surgical resection needs consideration in symptomatic patients [5-8]. Asymptomatic individuals require a long-range arrange and surgical operation as a prevention method to avoid complications [5]. In this case, the symptomatic individual received total surgical resection.

\section{Conclusions}

In conclusion, this case illustrated a unusual calcified right ventricular fibroma in the adult. Surgical excision is a effective means to improve prognosis in symptomatic patients.

\section{Declarations:}

\section{List of abbreviations}

TTE, Transthoracic echocardiography; CT, Cardiac computed tomography; TEE, transesophageal echocardiography; MRI, Magnetic resonance imaging;

\section{Ethics approval and consent to participate}

The study was approved by the Institutional Review Board at the First Affiliated Hospital of Zhejiang University (Hangzhou, China). The procedures were conducted according to the principles of the Helsinki Declaration.

\section{Consent for publication}

Written informed consent was obtained from the patient for publication of this case report and any accompanying images. A copy of the written consent is available for review by the Editor-in-Chief of this journal.Availability of data and materials

Not applicable.

\section{Competing interests}

The authors declare that they have no competing interests.

Funding This research received no specific grant from any funding agency in the public, commercial, or not-for-profit sectors.

\section{Authors' contributions}


HG wrote the manuscript. SY carried out the intraoperative transesophageal echocardiography. ZH carried out the echocardiography. ZL revised the manuscript. SW helped to perform the operate and conceived the study. All authors have read and approved the final manuscript.

Acknowledgments This study was supported by grants from Scientific Research Fund of Zhejiang Provincial Education Department (No. Y201738814).

\section{References}

[1] Burke A and Virmani R. Pediatric heart tumours. Cardiovasc Pathol 2008; 17:193-8.

[2] Unsal $\mathrm{H}$ and Ekici E. Conservative management of a left ventricle cardiac fibroma in an asymptomatic child patient. Turk Kardiyol Dern Ars 2015;43: 481-3.

[3] Tao TY, Yahyavi-Firouz-Abadi N, Singh GK, et al.Pediatric cardiac tumors: clinical and imaging features. Radiographics 2014; 34: 1031-46.

[4] Burke A and Tavora F. The 2015 WHO classification of tumors of the heart and pericardium. J Thorac Oncol 2016;11: 441-52.

[5] Zheng XJ and Song B. Left ventricle primary cardiac fibroma in an adult: A case report. Oncol Lett 2018 16:5463-65.

[6] Gowdamarajan A, Michler R. Therapy for primary cardiac tumors: is there a role for heart transplantation? Curr Opin Cardiol 2000; 15:121-6.

[7] BurkeAP, Rosado-de-ChristensonM, T empletonPA, et al. Cardiac fibroma: clinicopathologic correlates and surgical treatment. J Thorac Cardiovasc Surg 1994;108:862-70.

[8] Darwazah AK, Shoeb J, Eissa SS: Pedunculated endocardial left ventricular fibroma presenting with cerebral and bilateral peripheral embolization. Ann Thorac Surg 2010; 89(3):965-967.

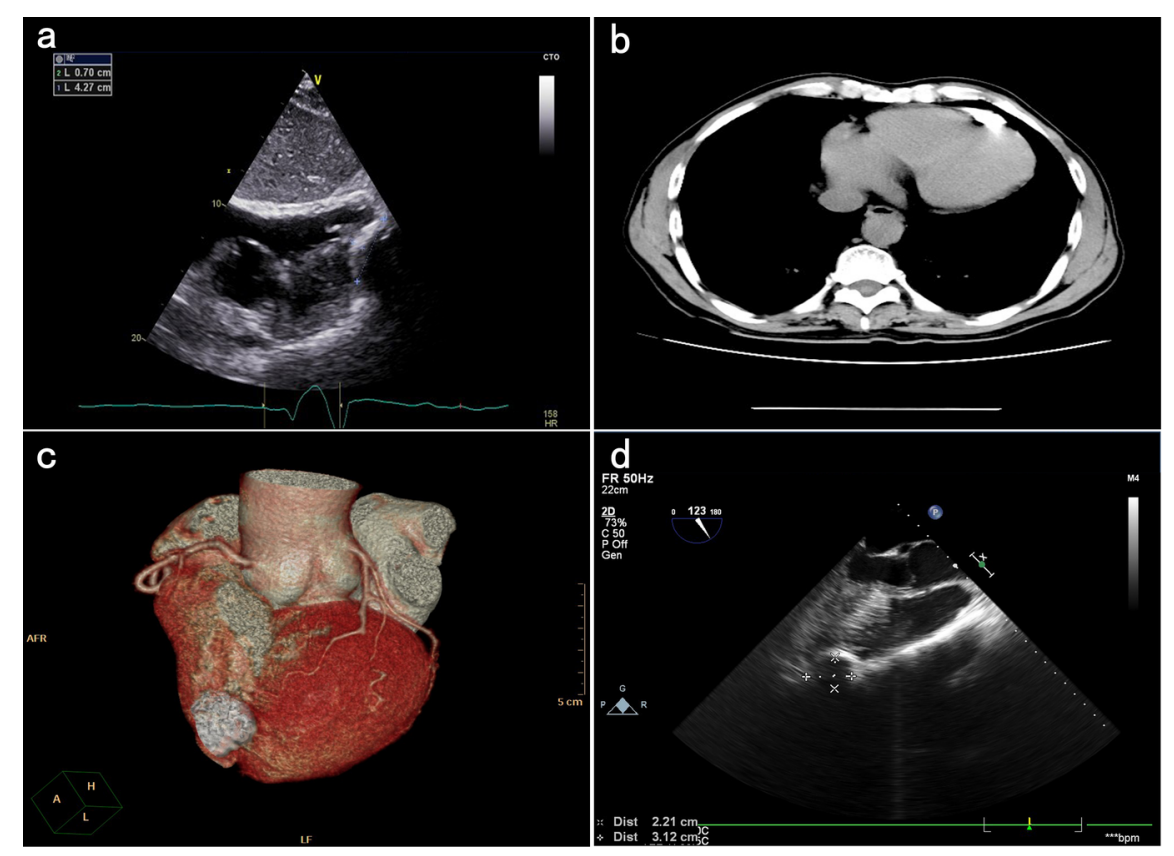



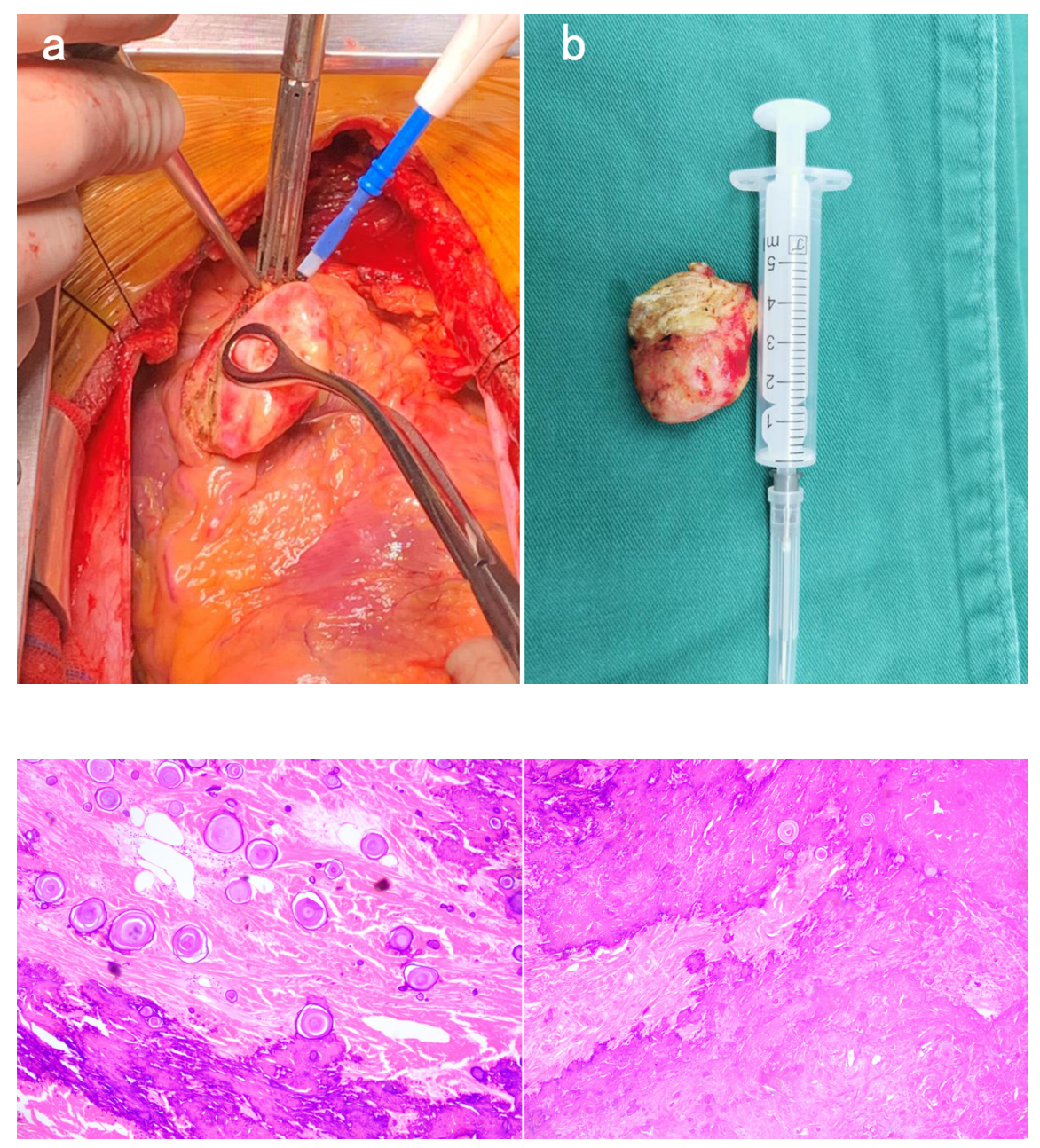